ПРИЛОЗИ, Одделение за природно-математички и биотехнички науки, МАНУ, том 38, бр. 2, стр. 153-164 (2017)

CONTRIBUTIONS, Section of Natural, Mathematical and Biotechnical Sciences, MASA, Vol. 38, No. 2, pp. 153-164 (2017)

Received: October 17, 2016

ISSN 1857-9027

Accepted: July 12, 2017

e-ISSN 1857-9949

UDC: $511.331: 517.581$

DOI: $10.20903 /$ csnmbs.masa.2017.38.2.111

Original scientific paper

\title{
CONVERGENCE OF DIRICHLET SERIES AND EULER PRODUCTS
}

\author{
Doug S. Phillips ${ }^{1}$, Peter Zvengrowski ${ }^{2}$ \\ ${ }^{1}$ Information Technologies, University of Calgary \\ ${ }^{2}$ Department of Mathematics and Statistics, University of Calgary \\ e-mail: phillips@ucalgary.ca, zvengrow@ucalgary.ca
}

The first part of this paper deals with Dirichlet series, and convergence theorems are proved that strengthen the classical convergence theorem as found e.g. in Serre's "A Course in Arithmetic." The second part deals with Euler-type products. A convergence theorem is proved giving sufficient conditions for such products to converge in the half-plane having real part greater than $1 / 2$. Numerical evidence is also presented that suggests that the Euler products corresponding to Dirichlet $L$-functions $L(s, \chi)$, where $\chi$ is a primitive Dirichlet character, converge in this half-plane.

Keywords: Dirichlet series, Euler products, $L$-functions, primitive Dirichlet character

\section{INTRODUCTION}

The general theme of this note is convergence. In Section 2 this is studied for Dirichlet series and in Sections 3-5 for infinite products, in particular for Euler products. For the Dirichlet series we more or less follow [11] and give the proof (cf. Theorem 2.7) that a series such as the "Euler-Dedekind" or "alternating" zeta function $\eta(s)=\Sigma_{n=1}^{\infty}(-1)^{n-1} n^{-s}$, in addition to converging absolutely for $\sigma>1$ (here as usual $s=\sigma+i t$ ), converges conditionally for $\sigma>0$. Theorem 2.9 is a refinement of Theorem 2.7, and Theorem 2.12 a further refinement which gives sufficient conditions for convergence for $\sigma>.5$.

Section 3 gives numerical data which suggests that the Euler product for the Dirichlet $L$-function $L(s, \chi)$ associated to a primitive $\bmod q$ character $\chi(q \geq 3)$, which is well known to converge absolutely for $\sigma>1$, also converges for $\sigma>.5$. Section 4 then presents some theory which gives sufficient conditions for Euler-type products to converge for $\sigma>.5$. Section 5 gives further numerical evidence which, combined with Theorem 4.3, strongly suggests such convergence for the $L$-functions being considered. Of course, since (as we shall see in Section 4) convergent infinite products cannot equal 0 , this would imply the Generalized Riemann Hypothesis for all these $L$-functions, namely each such $L$ function cannot have a zero if $\sigma>.5$ (cf. [6], [10]).

Section 6 gives further examples and concludes with a few questions.

\section{DIRICHLET SERIES}

We commence with a little "review" material to establish some conventions and notation. By a Dirichlet series we mean an infinite series

$$
f(s)=\sum_{n=1}^{\infty} \frac{a_{n}}{n^{s}}=\sum_{n=1}^{\infty} a_{n} n^{-s}, \quad a_{n} \in \mathbb{C} .
$$

A very familiar example is the case $a_{n}=1, f$ is then the Riemann zeta function $\zeta$. For $t=0$, i.e. for $s=\sigma \in \mathbb{R}$, it is proved in elementary calculus that $\zeta(\sigma)$ diverges for $\sigma=1$ and is absolutely convergent for $\sigma>1$. This is called the " $p$-test" (where $p=\sigma$ ) but should really be called the " $\zeta$-test." Another familiar example (again when $s=\sigma \in \mathbb{R}$ ) is the Euler-Dedekind function $\eta(\sigma)$ defined in Section 
1. It is proved in elementary calculus that this series converges for $\sigma>0$, where the convergence is conditional for $0<\sigma \leq 1$ and absolute for $1<\sigma$. In this section we shall prove that very similar results hold, with appropriate hypotheses on the coefficients $a_{n}$, for $s \in \mathbb{C}$, i.e. dropping the condition $t=0$.

From elementary complex analysis, for any $x \in \mathbb{R}^{+}$, one has $\left|x^{s}\right|=x^{\sigma}$. In particular $\left|n^{-s}\right|=$ $n^{-\sigma}$. Using this together with the $\zeta$-test gives the next result immediately.

2.1 Proposition : If $\left|a_{n}\right|$ is bounded then the Dirichlet series $\Sigma a_{n} n^{-s}$ is absolutely convergent for $\sigma>1$.

In particular this holds for $\zeta(s), \eta(s)$ and all $L$ functions $L(s, \chi):=\Sigma_{n=1}^{\infty} \chi(n) n^{-s}$ for any Dirichlet character $\chi$, indeed $\left|a_{n}\right| \in\{0,1\}$ for these functions. 2.2 Examples : The $\bmod 3$ character $\chi_{2}^{3}$ is defined by $\chi_{2}^{3}(n)=0,1,-1$ for $n$ congruent respectively to $0,1,2$ modulo 3 . One has $L\left(s, \chi_{2}^{3}\right)=$ $1-2^{-s}+4^{-s}-5^{-s}+7^{-s}-8^{-s} \ldots$. By the Leibniz alternating series test we see that both $\eta(s), L\left(s, \chi_{2}^{3}\right)$ converge (conditionally) along the real line $s=\sigma$ for $0<\sigma \leq 1$. The first objective of this section is to show that this remains true for all $t$, i.e. Dirichlet series such as in these two examples are convergent for $\sigma>0$, for all $t$. The treatment is very close to that of [11].

2.3 Lemma : Let $\alpha, \beta, \sigma \in \mathbb{R}, 0<\sigma, 0<\alpha<\beta$. Then $\left|e^{-\alpha s}-e^{-\beta s}\right| \leq \frac{|s|}{\sigma}\left(e^{-\alpha \sigma}-e^{-\beta \sigma}\right)$.

Proof: We have

$$
\begin{aligned}
& e^{-\alpha s}-e^{-\beta s}=s \int_{\alpha}^{\beta} e^{-u s} d u \\
& \text { hence }\left|e^{-\alpha s}-e^{-\beta s}\right| \leq|s| \int_{\alpha}^{\beta}\left|e^{-u s}\right| d u \\
& =|s| \int_{\alpha}^{\beta} e^{-u \sigma} d u=\frac{|s|}{\sigma}\left(e^{-\alpha \sigma}-e^{-\beta \sigma}\right) .
\end{aligned}
$$

2.4 Corollary : Set $\alpha=\log (m), \beta=\log (n)$, $0<m<n, \sigma>0$, then

$$
\left|m^{-s}-n^{-s}\right| \leq \frac{|s|}{\sigma}\left(m^{-\sigma}-n^{-\sigma}\right) .
$$

2.5 Lemma (Abel's summation formula) : Let $a_{k}, b_{k} \in \mathbb{C}, n \geq 1$, and set $A_{n}=a_{1}+\cdots+a_{n}$. Then

$$
\sum_{k=1}^{n} a_{k} b_{k}=A_{n} b_{n+1}-\sum_{k=1}^{n} A_{k}\left(b_{k+1}-b_{k}\right) \text {. }
$$

Proof: Let $A_{0}=0$. Then

$$
\begin{aligned}
\sum_{k=1}^{n} a_{k} b_{k} & =\sum_{k=1}^{n}\left(A_{k}-A_{k-1}\right) b_{k}= \\
& =\sum_{k=1}^{n} A_{k} b_{k}-\sum_{k=1}^{n} A_{k} b_{k+1}+A_{n} b_{n+1}
\end{aligned}
$$

which is the same as the right hand side of Abel's formula.

2.6 Corollary : The sum $\Sigma_{k=1}^{\infty} a_{k} b_{k}$ converges if both $\Sigma_{k=1}^{\infty} A_{k}\left(b_{k+1}-b_{k}\right)$ and $\left\{A_{n} b_{n+1}\right\}$ are convergent.
We remark that Abel's summation formula can be thought of as a discrete version of the familiar integration by parts formula from calculus. This should be clear by writing them side by side as $\begin{aligned} & \sum_{k=1}^{n} b_{k} a_{k}=A_{n} b_{n+1}-\sum_{k=1}^{n} A_{k}\left(b_{k+1}-b_{k}\right) \\ & \int u d v=v u-\int v d u \text {. } \\ & \text { Before turning to the first main theorem }\end{aligned}$ this section, we recall some standard facts about convergence of an infinite series of complex numbers $z_{n}$. The partial sums are written $S_{n}:=\Sigma_{k=1}^{n} z_{k}$, and one says that $\Sigma_{k=1}^{\infty} z_{k}=S$ if and only if $\lim _{n \rightarrow \infty} S_{n}$ exists and equals $S$. In this case the series is said to be convergent. A necessary condition for convergence is $z_{n} \rightarrow 0$ as $n \rightarrow \infty$. A necessary and sufficient condition, the Cauchy convergence criterion, is that for any given real number $\varepsilon>0$ there exists $N \in \mathbb{N}$ such that for all $m, n \geq N, \quad\left|S_{n}-S_{m}\right|<\varepsilon$.

We now turn our attention to $z_{n}=a_{n} n^{-s}$, i.e. the Dirichlet series $\Sigma_{n=1}^{\infty} a_{n} n^{-s}, a_{n}, s \in \mathbb{C}$. The notation $A_{n}=a_{1}+\ldots+a_{n}$ for such a Dirichlet series will be used henceforth.

2.7 Theorem : Consider $\Sigma_{n=1}^{\infty} a_{n} n^{-s}, a_{n} \in \mathbb{C}$. If $\left\{\left|A_{n}\right|\right\}$ is bounded then the series converges for $\sigma>0$.

Proof: We have $\left|A_{n}\right| \leq C$, for some $C>0$ and for all $n$. We shall use Corollary 2.6, with $a_{n}=a_{n}$ and $b_{n}=n^{-s}$. Then $\left|A_{n} b_{n+1}\right|=\left|A_{n}\right| \cdot\left|b_{n+1}\right| \leq$ $C \cdot(n+1)^{-\sigma} \rightarrow 0$ as $n \rightarrow \infty$. Hence the second condition of Corollary 2.6, $\left\{A_{n} b_{n+1}\right\}$ converges (in this case to 0$)$, is satisfied.

For the first condition, we apply the Cauchy convergence criterion to $\sum_{k=1}^{\infty} A_{k}\left((k+1)^{-s}-k^{-s}\right)$. Given $\varepsilon>0$ and using Corollary 2.4 we have

$$
\begin{aligned}
\left|S_{n}-S_{m}\right| & =\left|\sum_{k=m+1}^{n} A_{k}\left((k+1)^{-s}-k^{-s}\right)\right| \\
& \leq C \cdot \sum_{k=m+1}^{n}\left|(k+1)^{-s}-k^{-s}\right| \\
& \leq \frac{C|s|}{\sigma} \sum_{k=m+1}^{n}\left(\frac{1}{k^{\sigma}}-\frac{1}{(k+1)^{\sigma}}\right) \\
& =\frac{C|s|}{\sigma}\left(\frac{1}{(m+1)^{\sigma}}-\frac{1}{(n+1)^{\sigma}}\right) \\
& \leq \frac{C|s|}{\sigma(m+1)^{\sigma}}<\varepsilon
\end{aligned}
$$

for $m$ sufficiently large.

The first objective of this section is thus accomplished. We give a corollary. Recall that the trivial (also called principal) Dirichlet character modulo $q$ is given by $\chi(n)=0$ for all $n$ such that $\operatorname{gcd}(q, n)>1$, and $\chi(n)=1$ when $\operatorname{gcd}(q, n)=1$.

2.8 Corollary : For $\eta(s)$ or for $L(s, \chi)$ with $\chi$ any non-trivial Dirichlet character $\chi$ modulo $q$, the Dirichlet series converges for $\sigma>0$.

Proof: For $\eta, A_{n} \in\{0,1\}$ is bounded. For any non-trivial character $\chi$ modulo $q$ one has $A_{q}=$ $\chi(1)+\ldots+\chi(q)=0$ (cf. [10] Ex. 2.2.8) so $\left\{\left|A_{n}\right|\right\}$ is periodic modulo $q$, hence finite and bounded. 
We remark that Theorem 2.7 is proved in [11], but the proof is a little less direct than the one given above, and is restricted to the case $a_{n} \in \mathbb{R}$ (for no apparent reason). We also remark that Corollary 2.8 is identical to [10] Ex. 2.3.4.

The second objective of this section is to consider possible strengthening of the above results, in particular 2.3, 2.7, and their corollaries. It will be seen in Sections 4-5 that such strengthening could be very useful. First consider Corollary 2.4. Another obvious (second) upper bound is $\left|m^{-s}-n^{-s}\right| \leq$ $\left|m^{-s}\right|+\left|n^{-s}\right|=m^{-\sigma}+n^{-\sigma}$. It can be seen that for each fixed values for $m, n, \sigma$ there is a $t_{*}$ such that the first upper bound (from 2.4) is better for $t<t_{*}$ whereas the second, which is simply a constant, is better for $t>t_{*}$. Indeed the second becomes better and better as $t$ increases. Whether this can be used in some way to strengthen Theorem 2.7 is presently not known. It may also be possible to find a third upper bound that improves both the first and second (of course their minimum will be one such) and also can be used to strengthen 2.7.

It is in fact possible to strengthen Theorem 2.7 using Corollary 2.4 as it stands, and the next two theorems are examples.
2.9 Theorem : Consider $\Sigma_{n=1}^{\infty} a_{n} n^{-s}, a_{n} \in \mathbb{C}$. If there exists a constant $C>0$ such that $\left|A_{n}\right|<$ $C \cdot \log (n), n \geq 2$, then the series converges for $\sigma>0$. Proof: As in the proof of Theorem 2.7, the second convergence condition follows since $C \cdot \log (n) \cdot(n+$ $1)^{-\sigma} \rightarrow 0$ as $n \rightarrow \infty$. For the first convergence condition, proceeding as in 2.7 , we have

$$
\begin{aligned}
\left|S_{n}-S_{m}\right| & =\left|\sum_{k=m+1}^{n} A_{k} \cdot\left((k+1)^{-s}-k^{-s}\right)\right| \\
& \leq \sum_{k=m+1}^{n}\left|A_{k}\right| \cdot\left|k^{-s}-(k+1)^{-s}\right| .
\end{aligned}
$$

Here $m \geq 1, k \geq 2$, hence from both the hypothesis and Corollary 2.4

$$
\begin{aligned}
& \sum_{k=m+1}^{n}\left|A_{k}\right| \cdot\left|k^{-s}-(k+1)^{-s}\right| \\
& \leq \frac{C|s|}{\sigma} \sum_{k=m+1}^{n} \log (k) \cdot\left(k^{-\sigma}-(k+1)^{-\sigma}\right) .
\end{aligned}
$$

For convenience write $C|s| / \sigma=K$ henceforth, then the last expression, after a small rearrangement of the terms, equals

$$
\begin{array}{r}
\left.K\left[\log (m+1) \cdot(m+1)^{-\sigma}+\sum_{k=m+1}^{n-1}(\log (k+1)-\log (k)) \cdot(k+1)^{-\sigma}-\log (n) \cdot(n+1)^{-\sigma}\right)\right] \\
=K\left[\log (m+1) \cdot(m+1)^{-\sigma}-\log (n) \cdot(n+1)^{-\sigma}+\sum_{k=m+1}^{n-1} \log \left(1+\frac{1}{k}\right) \cdot(k+1)^{-\sigma}\right] .
\end{array}
$$

Next note that for $0 \leq u \leq 1, \log (1+u)=u-u^{2} / 2+u^{3} / 3+\ldots=u+\beta_{u}$, where $\left|\beta_{u}\right| \leq u^{2} / 2$, as in the Leibniz convergence test for series with alternating signs (in fact, using the mean value theorem from elementary calculus, one sees that this remains true for $0 \leq u$ ). The previous sum thus equals

$$
\begin{gathered}
K \cdot\left[\frac{\log (m+1)}{(m+1)^{\sigma}}-\frac{\log (n)}{(n+1)^{\sigma}}+\sum_{k=m+1}^{n-1}\left(\frac{1}{k}+\beta_{1 / k}\right) \cdot(k+1)^{-\sigma}\right] \\
\quad \leq K \cdot\left[\frac{\log (m+1)}{(m+1)^{\sigma}}+\sum_{k=m+1}^{\infty} k^{-1-\sigma}+\frac{1}{2} \sum_{k=m+1}^{\infty} k^{-2-\sigma}\right] .
\end{gathered}
$$

For $\sigma>0$ the two summations are absolutely convergent, so given $\varepsilon>0$, taking $m$ sufficiently large will clearly guarantee that each of the three terms in the above formula will be smaller than $\varepsilon /(3 K)$, completing the proof.

Since the derivative of $n^{s}$ is $\log (n) \cdot n^{s}$, we can use Theorem 2.9 to obtain a corollary similar to 2.8 . 2.10 Corollary : For $f(s)=\eta(s)$ or for $f(s)=$ $L(s, \chi)$ with $\chi$ any non-trivial Dirichlet character modulo $q$, the Dirichlet series for $f^{\prime}(s)$ converges for $\sigma>0$.

We next prove a convergence theorem even stronger than Theorem 2.11, but this time with the additional hypothesis that $\sigma>.5$. One easy lemma is first needed.

2.11 Lemma : For $k>0,(k+1)^{1 / 2}-k^{1 / 2}$ $=\frac{1}{2 k^{1 / 2}}+\beta_{k}$, where $\left|\beta_{k}\right|<\frac{1}{8 k^{3 / 2}}$.

Proof: This is almost immediate from the binomial theorem, which gives the convergent series

$$
\beta_{k}=-\frac{1}{8 k^{3 / 2}}+\frac{3}{2^{3} \cdot 3 ! \cdot k^{5 / 2}}-\frac{3 \cdot 5}{2^{4} \cdot 4 ! \cdot k^{7 / 2}}+\ldots .
$$

The Leibniz test for alternating series completes the proof.

2.12 Theorem : Consider $\sum_{n=1}^{\infty} a_{n} n^{-s}, a_{n} \in \mathbb{C}$. If there exists a constant $C>0$ such that $\left|A_{n}\right|<$ $C \cdot n^{1 / 2}, n \geq 1$, then the series converges for $\sigma>1 / 2$. 
Proof : The proof is similar to the proofs of both 2.7 and 2.9, using the Lemma 2.11. The second convergence condition of Corollary 2.6 follows since $C \cdot n^{1 / 2} \cdot(n+1)^{-\sigma} \rightarrow 0$ as $n \rightarrow \infty$.

For the first convergence condition, proceeding as in 2.7, 2.9, and omitting the first few steps (which are identical), we have

$$
\left|S_{n}-S_{m}\right| \leq \frac{C|s|}{\sigma} \sum_{k=m+1}^{n} k^{1 / 2} \cdot\left(k^{-\sigma}-(k+1)^{-\sigma}\right) \text {. }
$$

For convenience write $C|s| / \sigma=K$ henceforth, then the last expression, after a small rearrangement of the terms, equals

$$
\begin{aligned}
& K\left[(m+1)^{1 / 2} \cdot(m+1)^{-\sigma}\right. \\
& \left.\left.+\sum_{k=m+1}^{n-1}\left((k+1)^{1 / 2}-k^{1 / 2}\right) \cdot(k+1)^{-\sigma}-n^{1 / 2} \cdot(n+1)^{-\sigma}\right)\right] .
\end{aligned}
$$

Now using Lemma 2.11, this equals

$$
\begin{gathered}
K \cdot\left[\frac{(m+1)^{1 / 2}}{(m+1)^{\sigma}}-\frac{n^{1 / 2}}{(n+1)^{\sigma}}+\sum_{k=m+1}^{n-1}\left(\frac{1}{2 \cdot k^{1 / 2}}+\beta_{k}\right) \cdot(k+1)^{-\sigma}\right], \text { where }\left|\beta_{k}\right|<\frac{1}{8 k^{3 / 2}} \\
\leq K \cdot\left[\frac{(m+1)^{1 / 2}}{(m+1)^{\sigma}}+\frac{1}{2} \sum_{k=m+1}^{\infty} k^{-1 / 2-\sigma}+\frac{1}{8} \sum_{k=m+1}^{\infty} k^{-3 / 2-\sigma}\right]
\end{gathered}
$$

For $\sigma>1 / 2$ the two summations are absolutely convergent, so given $\varepsilon>0$, taking $m$ sufficiently large will clearly guarantee that each of the three terms in the above formula will be smaller than $\varepsilon /(3 K)$, completing the proof.

\section{CONVERGENCE OF EULER PRODUCTS FOR $\sigma>.5$}

In this section we simply present some numerical evidence for convergence of certain Euler products in the half plane $\sigma>.5$. The Euler products for any Dirichlet $L$-function and the Riemann zeta function are well known to converge absolutely for $\sigma>1$. We now give some numerical evidence here that for an $L$-function coming from a primitive character $\chi$ $\bmod q, q \geq 3$, the Euler product

$$
L(s, \chi)=\prod_{p} \frac{1}{1-\chi(p) \cdot p^{-s}},
$$

where the product is taken over all primes $p$, converges for $\sigma>.5$ and diverges for smaller $\sigma$. Three primitive characters are considered, $\chi_{2}^{3}$ which takes values $0,1,-1$ as $n$ is respectively congruent to $0,1,2$ modulo $3, \chi_{2}^{4}$ which takes values $0,1,0,-1$ as $n$ is respectively congruent to $0,1,2,3$ modulo 4 , and $\chi_{2}^{5}$ which takes values $0,1, i,-i,-1$ as $n$ is respectively congruent to $0,1,2,3,4$ modulo 5 . We consider $s=\sigma+30 i$, for $\sigma=.4$ (showing divergence) and for $\sigma=.55, .6, .7, .8, .9,1.0,1.1,1.5$, which show stronger and stronger convergence as $\sigma$ increases. Of course for $\sigma=1.1,1.5$ convergence is known and is absolute. We choose $t=30$ as a fairly typical $t$ value, similar results can be seen for other $t$ values.

The figures below show the absolute value of the truncation error $\Delta$ for three Dirichlet $L$-functions as a function of the number of factors taken in their Euler product representations. Curves are shown for selected values of $\sigma$. To aid in extracting numerical values from the graphs, below each figure is a corresponding table giving $\Delta$ when the number of factors is a power of 10 .

Preliminary calculations were performed with Maple [9], which also provided reference values for the $L$-functions used in calculating the truncation errors. To overcome performance limitations of Maple when extending the results to large numbers of factors in the Euler products, a Fortran program based on MPFUN2015 libraries [2] was used. Mathematica [12] was used to generate a file of the first $10^{9}$ primes, which was used as input to the Fortran calculation. Thanks go to Information Technologies at the University of Calgary for providing access to the large-memory nodes of the Helix cluster for the bulk of the computations. 


\section{Euler product truncation error vs. number of factors Dirichlet L-function $L\left(\sigma+30 i, \chi_{2}^{3}\right)$}

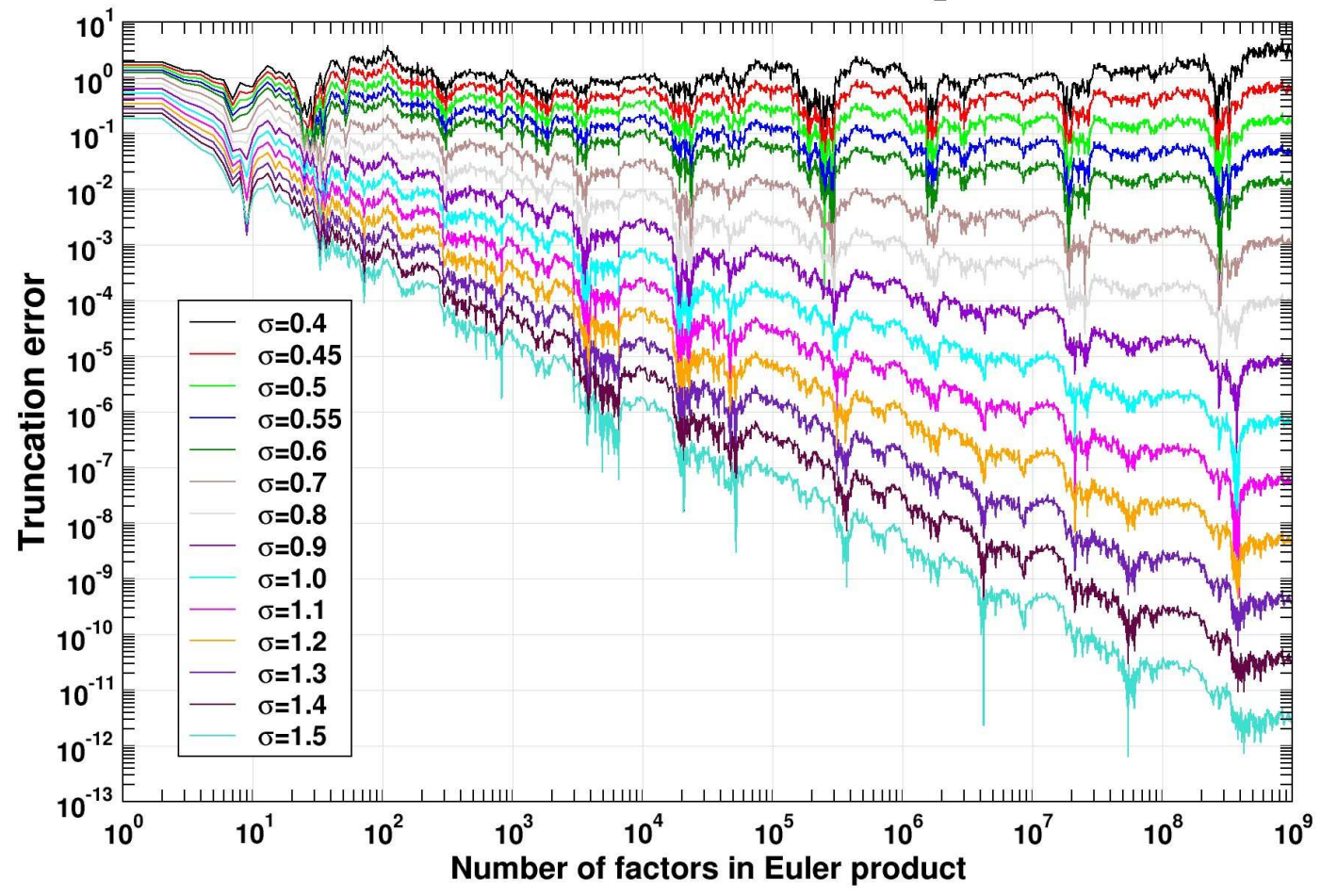

Figure 1. Graph of error terms for $L\left(\sigma+30 i, \chi_{2}^{3}\right)$

\begin{tabular}{|c|c|c|c|c|c|c|c|c|}
\hline \hline$\sigma$ & $\Delta\left(10^{2}\right)$ & $\Delta\left(10^{3}\right)$ & $\Delta\left(10^{4}\right)$ & $\Delta\left(10^{5}\right)$ & $\Delta\left(10^{6}\right)$ & $\Delta\left(10^{7}\right)$ & $\Delta\left(10^{8}\right)$ & $\Delta\left(10^{9}\right)$ \\
\hline \hline .4 & 2.64 & .948 & 1.04 & 1.53 & 1.28 & .929 & 1.49 & 3.16 \\
\hline .55 & .596 & .217 & .196 & .135 & .0809 & .0595 & .0513 & .0497 \\
\hline .6 & .384 & .131 & .107 & .0628 & .0335 & .0222 & .0166 & .0141 \\
\hline .7 & .167 & .0478 & .0313 & .0139 & .00582 & .00304 & .00175 & .00116 \\
\hline .8 & .0757 & .0175 & .00909 & .00311 & .00102 & $4.14 \times 10^{-4}$ & $1.85 \times 10^{-4}$ & $9.57 \times 10^{-5}$ \\
\hline .9 & .0352 & .00646 & .00265 & $7.02 \times 10^{-4}$ & $1.80 \times 10^{-4}$ & $5.66 \times 10^{-5}$ & $1.97 \times 10^{-5}$ & $7.98 \times 10^{-6}$ \\
\hline 1.0 & .0167 & .00239 & $7.74 \times 10^{-4}$ & $1.59 \times 10^{-4}$ & $3.18 \times 10^{-5}$ & $7.73 \times 10^{-6}$ & $2.10 \times 10^{-6}$ & $6.69 \times 10^{-7}$ \\
\hline 1.1 & .00801 & $8.89 \times 10^{-4}$ & $2.28 \times 10^{-4}$ & $3.63 \times 10^{-5}$ & $5.65 \times 10^{-6}$ & $1.06 \times 10^{-6}$ & $2.24 \times 10^{-7}$ & $5.65 \times 10^{-8}$ \\
\hline 1.5 & $4.63 \times 10^{-4}$ & $1.76 \times 10^{-5}$ & $1.76 \times 10^{-6}$ & $1.02 \times 10^{-7}$ & $5.84 \times 10^{-9}$ & $3.70 \times 10^{-10}$ & $2.99 \times 10^{-11}$ & $3.00 \times 10^{-12}$ \\
\hline \hline
\end{tabular}

TABLE 1. Error terms for $L\left(\sigma+30 i, \chi_{2}^{3}\right)$ 


\section{Euler product truncation error vs. number of factors Dirichlet L-function $L\left(\sigma+30 i, \chi_{2}^{4}\right)$}

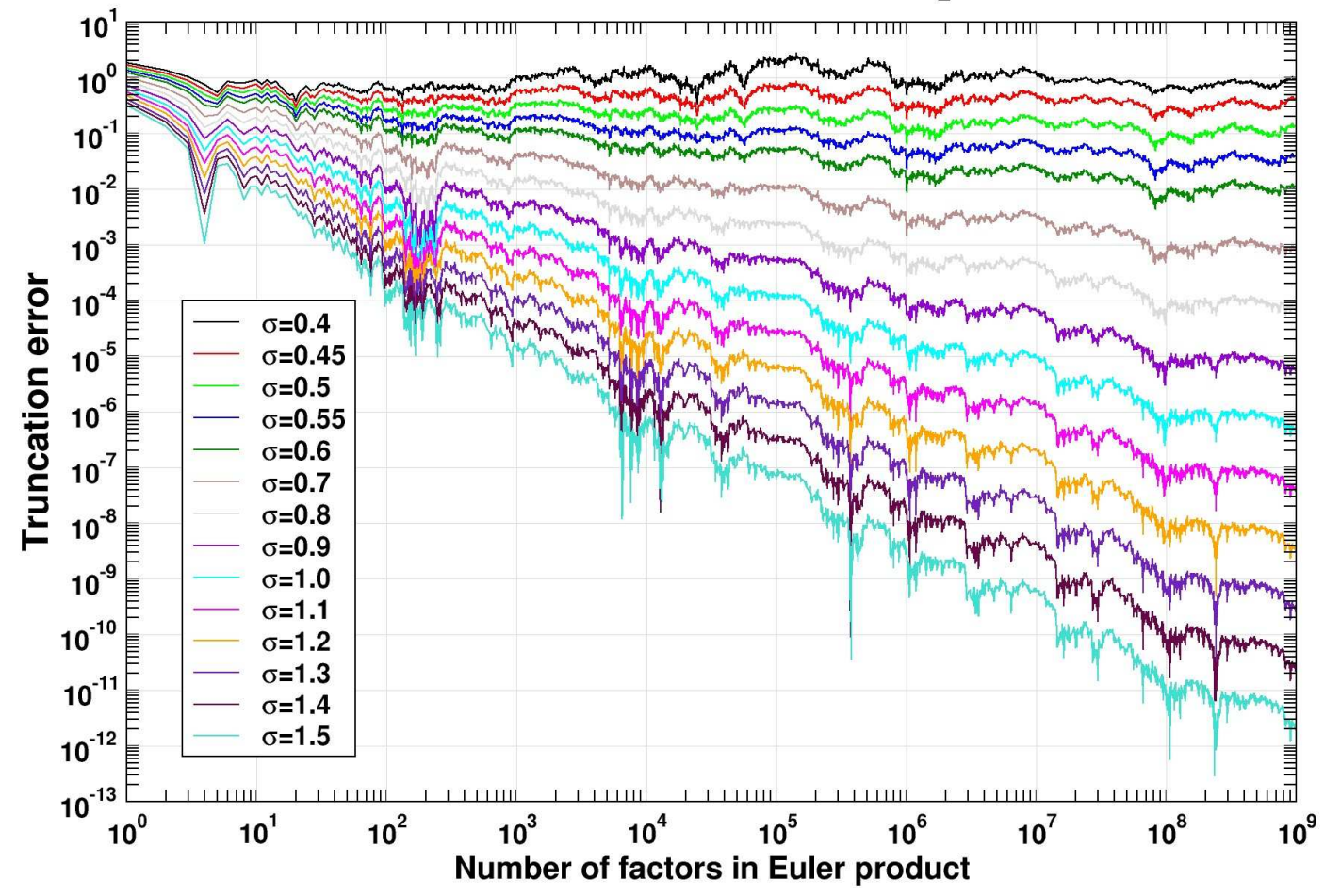

Figure 2. Graph of error terms for $L\left(\sigma+30 i, \chi_{2}^{4}\right)$

\begin{tabular}{|c|c|c|c|c|c|c|c|c|}
\hline \hline$\sigma$ & $\Delta\left(10^{2}\right)$ & $\Delta\left(10^{3}\right)$ & $\Delta\left(10^{4}\right)$ & $\Delta\left(10^{5}\right)$ & $\Delta\left(10^{6}\right)$ & $\Delta\left(10^{7}\right)$ & $\Delta\left(10^{8}\right)$ & $\Delta\left(10^{9}\right)$ \\
\hline \hline .4 & .599 & .970 & 1.43 & 1.84 & .573 & 1.21 & .673 & .979 \\
\hline .55 & .154 & .194 & .118 & .109 & .0315 & .0642 & .0253 & .0391 \\
\hline .6 & .0998 & .115 & .0569 & .0487 & .0122 & .0236 & .00756 & .0111 \\
\hline .7 & .0427 & .0414 & .0140 & .0104 & .00197 & .00327 & $6.75 \times 10^{-4}$ & $8.84 \times 10^{-4}$ \\
\hline .8 & .0187 & .0151 & .00353 & .00229 & $3.40 \times 10^{-4}$ & $4.62 \times 10^{-4}$ & $6.08 \times 10^{-5}$ & $7.19 \times 10^{-5}$ \\
\hline .9 & .00835 & .00557 & .000915 & .000515 & $6.15 \times 10^{-5}$ & $6.62 \times 10^{-5}$ & $5.56 \times 10^{-6}$ & $5.96 \times 10^{-6}$ \\
\hline 1.0 & .00379 & .00207 & .000243 & .000117 & $1.14 \times 10^{-5}$ & $9.53 \times 10^{-6}$ & $5.19 \times 10^{-7}$ & $4.99 \times 10^{-7}$ \\
\hline 1.1 & .00175 & .000774 & $6.63 \times 10^{-5}$ & $2.66 \times 10^{-5}$ & $2.14 \times 10^{-6}$ & $1.38 \times 10^{-6}$ & $5.03 \times 10^{-8}$ & $4.22 \times 10^{-8}$ \\
\hline 1.5 & $8.87 \times 10^{-5}$ & $1.57 \times 10^{-5}$ & $5.22 \times 10^{-7}$ & $7.32 \times 10^{-8}$ & $2.66 \times 10^{-9}$ & $6.10 \times 10^{-10}$ & $7.12 \times 10^{-12}$ & $2.31 \times 10^{-12}$ \\
\hline \hline
\end{tabular}

TABLE 2. Error terms for $L\left(\sigma+30 i, \chi_{2}^{4}\right)$ 


\section{Euler product truncation error vs. number of factors Dirichlet L-function $\mathrm{L}\left(\sigma+30 \mathrm{i}, \chi_{2}^{5}\right)$}

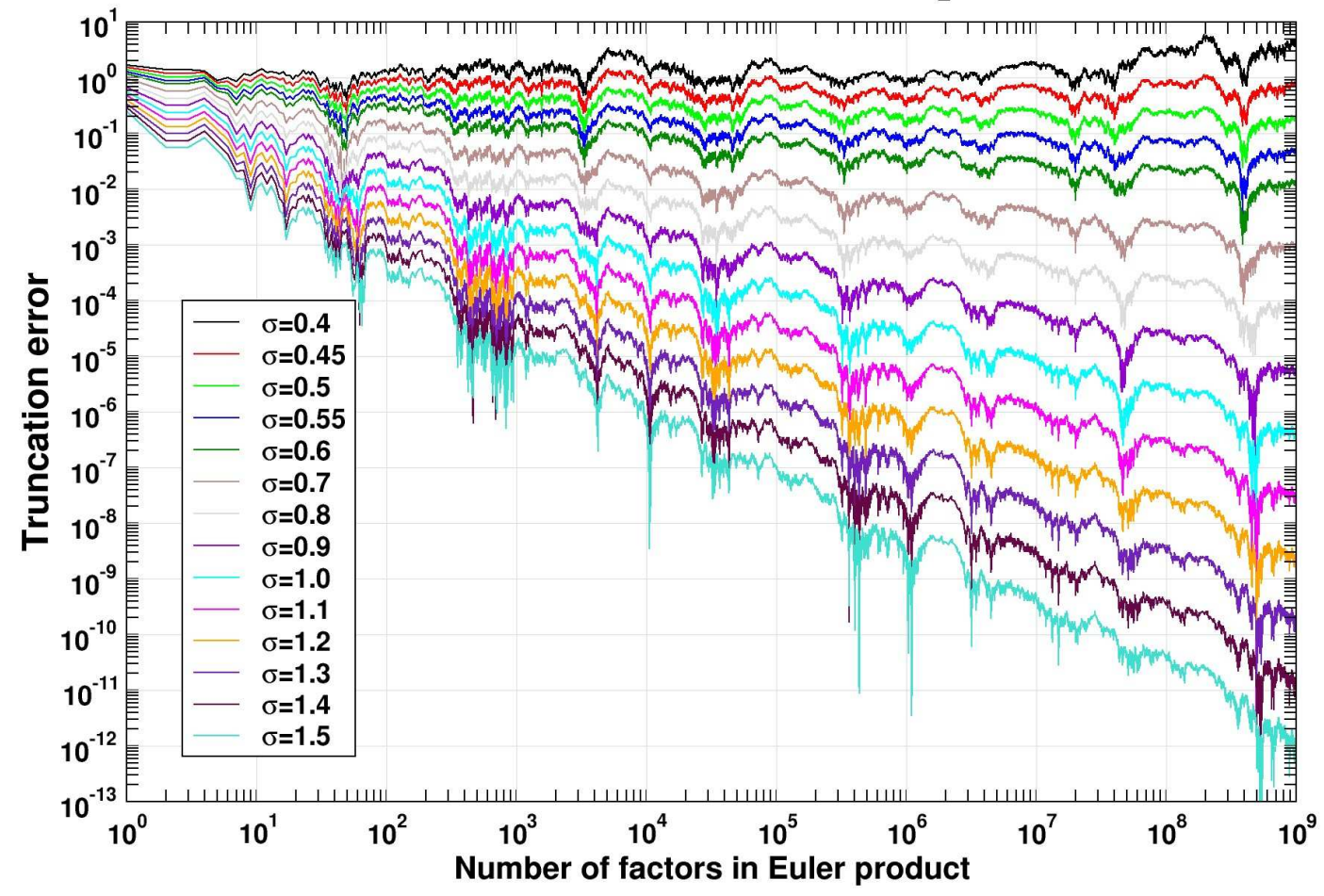

Figure 3. Graph of error terms for $L\left(\sigma+30 i, \chi_{2}^{5}\right)$

\begin{tabular}{|c|c|c|c|c|c|c|c|c|}
\hline \hline$\sigma$ & $\Delta\left(10^{2}\right)$ & $\Delta\left(10^{3}\right)$ & $\Delta\left(10^{4}\right)$ & $\Delta\left(10^{5}\right)$ & $\Delta\left(10^{6}\right)$ & $\Delta\left(10^{7}\right)$ & $\Delta\left(10^{8}\right)$ & $\Delta\left(10^{9}\right)$ \\
\hline \hline .4 & 1.40 & 1.75 & 1.49 & 1.80 & .767 & 1.86 & 2.80 & 3.95 \\
\hline .55 & .452 & .283 & .161 & .190 & .0577 & .0977 & .0756 & .0476 \\
\hline .6 & .310 & .163 & .0784 & .0871 & .0231 & .0347 & .0241 & .0130 \\
\hline .7 & .145 & .0556 & .0191 & .0185 & .00367 & .00439 & .00248 & $9.94 \times 10^{-4}$ \\
\hline .8 & .0686 & .0195 & .00473 & .00398 & $5.86 \times 10^{-4}$ & $5.64 \times 10^{-4}$ & $2.58 \times 10^{-4}$ & $7.66 \times 10^{-5}$ \\
\hline .9 & .0325 & .00693 & .00120 & $8.74 \times 10^{-4}$ & $9.45 \times 10^{-5}$ & $7.38 \times 10^{-5}$ & $2.73 \times 10^{-5}$ & $5.94 \times 10^{-6}$ \\
\hline 1.0 & .0155 & .00249 & $3.08 \times 10^{-4}$ & $1.94 \times 10^{-4}$ & $1.54 \times 10^{-5}$ & $9.82 \times 10^{-6}$ & $2.90 \times 10^{-6}$ & $4.62 \times 10^{-7}$ \\
\hline 1.1 & .00741 & .000901 & $8.13 \times 10^{-5}$ & $4.34 \times 10^{-5}$ & $2.55 \times 10^{-6}$ & $1.33 \times 10^{-6}$ & $3.11 \times 10^{-7}$ & $3.60 \times 10^{-8}$ \\
\hline 1.5 & $4.05 \times 10^{-4}$ & $1.63 \times 10^{-5}$ & $5.50 \times 10^{-7}$ & $1.16 \times 10^{-7}$ & $2.41 \times 10^{-9}$ & $5.01 \times 10^{-10}$ & $4.33 \times 10^{-11}$ & $1.34 \times 10^{-12}$ \\
\hline \hline
\end{tabular}

TABLE 3. Error terms for $L\left(\sigma+30 i, \chi_{2}^{5}\right)$

\section{THEORY OF EULER PRODUCT CONVERGENCE}

We start this section with a brief discussion of infinite products and the related convergence issues, and conclude with a theorem that seems to give an approach to proving that Euler products of the type considered in Section 3 converge, for $\sigma>1 / 2$. Intuitively one would say that $\Pi_{n=1}^{\infty} u_{n}, u_{n} \in \mathbb{C}$, converges when $\lim _{N \rightarrow \infty} \Pi_{n=1}^{N} u_{n}=L$ exists, and then define $\Pi_{n=1}^{\infty} u_{n}=L$. But this has complications, especially if any $u_{n}=0$. For the most general definition see Apostol's text [1], p. 207. This definition has quite a few cases and even a few surprises, e.g. if $u_{n}=1 / n$ we say $\Pi_{n=1}^{\infty} u_{n}$ diverges to 0 . For our purposes it suffices to avoid these complications by using a subset of the Apostol definition and requiring:

(a) $u_{n} \neq 0$ for all $n$, and

(b) $\lim _{n \rightarrow \infty} u_{n}=1$.

Then we may now follow Lang [8] pp. 372-373 and define $\Pi_{n=1}^{\infty} u_{n}$ to be convergent (resp. absolutely convergent) if the infinite series $\Sigma_{n=1}^{\infty} \log \left(u_{n}\right)$ is convergent (resp. absolutely convergent), provided 
we are a little careful with the multivalued logarithmic function, as follows. From (a) $\log \left(u_{n}\right)$ is defined for all $n$, and from (b), discarding a finite number of terms if so required (which has no effect on convergence issues), we can suppose $\left|u_{n}-1\right|<1 / 2$ for all sufficiently large $n$. We then choose the branch of the logarithm for which $\log (1)=0$. This also implies $\lim _{n \rightarrow \infty}\left(\log \left(u_{n}\right)\right)=0$. Now write $w:=\sum_{n=1}^{\infty} \log \left(u_{n}\right) \in \mathbb{C}$, and using the continuity of the exponential function we see that

$$
\begin{aligned}
\prod_{n=1}^{\infty} u_{n}=\lim _{N \rightarrow \infty} & \prod_{n=1}^{N} u_{n}=\lim _{N \rightarrow \infty} e^{\left(\sum_{n=1}^{N} \log \left(u_{n}\right)\right)} \\
& =e^{\lim _{N \rightarrow \infty}\left(\sum_{n=1}^{N} \log \left(u_{n}\right)\right)}=e^{w}
\end{aligned}
$$

exists and further is non-zero. It is also clear that $\Pi_{n=1}^{\infty}\left(u_{n}\right)^{-1}=1 /\left(e^{w}\right)$.

We shall henceforth write $u_{n}=1-\alpha_{n}$, and next give two results that connect the convergence of $\Pi_{n=1}^{\infty} u_{n}$ with the convergence of $\Sigma_{n=1}^{\infty} \alpha_{n}$. The first concerns absolute convergence and is found in many texts, cf. [8]. The second concerns convergence and can be found in [3], p.405, at least for the case $u_{n} \in \mathbb{R}$.

4.1 Theorem : Let $\alpha_{n} \in \mathbb{C} \backslash\{1\}$ and suppose $\Sigma_{n=1}^{\infty} \alpha_{n}$ is absolutely convergent. Then $\Pi_{n=1}^{\infty} u_{n}$ is absolutely convergent.

Sketch of proof (following [8]): The hypotheses on $\alpha_{n}$ imply conditions (a), (b) for $u_{n}$ hold, so we consider $\Sigma_{n=1}^{\infty} \log \left(u_{n}\right)=\Sigma_{n=1}^{\infty} \log \left(1-\alpha_{n}\right)$. Discarding a finite number of $\alpha_{n}$ if necessary we have $\left|\alpha_{n}\right|<1 / 2$, whence

$$
\begin{gathered}
\log \left(1-\alpha_{n}\right)=-\alpha_{n}-\frac{\alpha_{n}^{2}}{2}-\frac{\alpha_{n}^{3}}{3}-\ldots= \\
-\alpha_{n}\left(1+\frac{\alpha_{n}}{2}+\frac{\alpha_{n}^{2}}{3}+\ldots\right) .
\end{gathered}
$$

It is then easily seen that $\left|\log \left(1-\alpha_{n}\right)\right| \leq$ $(3 / 2) \cdot\left|\alpha_{n}\right|$ and the convergence of $\Sigma_{n=1}^{\infty}\left|\alpha_{n}\right|$ thus implies convergence of $\Sigma_{n=1}^{\infty}\left|\log \left(u_{n}\right)\right|$.

The proofs of the next lemma and theorem follow Bartle's proofs for the case $\alpha_{n} \in \mathbb{R}$ (cf. [3], given as a "Project"), with a couple of changes that are discussed in Remark 4.5 below.

4.2 Lemma : Let $z \in \mathbb{C},|z|<1 / 2$. Then $(1 / 6)|z|^{2}<|z+\log (1-z)|<(5 / 6)|z|^{2}$.

Proof: We have

$z+\log (1-z)=z-z-\frac{z^{2}}{2}-\frac{z^{3}}{3}-\ldots=-\frac{z^{2}}{2}(1+R)$, where $R=\sum_{n=1}^{\infty} \frac{2 z^{n}}{n+2}$. Now $|R| \leq(2 / 3)|z|+$ $(2 / 4)|z|^{2}+(2 / 5)|z|^{3}+\ldots \leq(2 / 3)|z|\left(1+|z|+|z|^{2}+\ldots\right)$ $=\frac{2|z|}{3} \frac{1}{1-|z|}<\frac{2}{3} \cdot \frac{1}{2} \cdot \frac{1}{1-1 / 2}=\frac{2}{3}$, whence $1 / 3<$ $|1+R|<5 / 3$. Multiplying by $|z|^{2} / 2$ completes the proof.
4.3 Theorem : Let $\alpha_{n} \in \mathbb{C} \backslash\{1\}$ and suppose $\Sigma_{n=1}^{\infty} \alpha_{n}$ is convergent. Then $\Pi_{n=1}^{\infty} u_{n}$ is convergent if $\sum_{n=1}^{\infty}\left|\alpha_{n}\right|^{2}$ is convergent.

Proof: We start as in the proof of 4.1 and have (again $n$ is assumed sufficiently large so $\left|\alpha_{n}\right|<1 / 2$ ) $\log \left(1-\alpha_{n}\right)=-\alpha_{n}+\beta_{n}$, where $\beta_{n}=\alpha_{n}+\log \left(1-\alpha_{n}\right)$, so by Lemma $4.2(1 / 6)\left|\alpha_{n}\right|^{2}<\left|\beta_{n}\right|<(5 / 6)\left|\alpha_{n}\right|^{2}$. By hypothesis $\Sigma_{n=1}^{\infty} \alpha_{n}$ converges, thus $\Sigma_{n=1}^{\infty} \log \left(1-\alpha_{n}\right)$ converges if and only if $\Sigma_{n=1}^{\infty} \beta_{n}$ converges. But by the above inequality (right-hand side) this will follow from the convergence of $\Sigma_{n=1}^{\infty}\left|\alpha_{n}\right|^{2}$, indeed $\Sigma_{n=1}^{\infty} \beta_{n}$ is absolutely convergent here.

4.4 Corollary: Let $\alpha_{n} \in \mathbb{C} \backslash\{1\}$ and suppose $\sum_{n=1}^{\infty} \alpha_{n}$ is convergent. Then $\Pi_{n=1}^{\infty} u_{n}$ is convergent if $\alpha_{n}=O\left(n^{-r}\right), r>1 / 2$.

4.5 Remark: In the real case $\alpha_{n} \in \mathbb{R}$, as in [3], one actually obtains the following stronger result: Let $\alpha_{n} \in \mathbb{R}, \alpha_{n}<1$, and suppose $\Sigma_{n=1}^{\infty} \alpha_{n}$ is convergent. Then $\Pi_{n=1}^{\infty} u_{n}$ is convergent if and only if $\Sigma_{n=1}^{\infty}\left|\alpha_{n}\right|^{2}$ is convergent. To see this one simply observes, for $n$ sufficiently large, that $\beta_{n}=-\frac{\alpha_{n}^{2}}{2}-\frac{\alpha_{n}^{3}}{3}-\ldots=$ $-\frac{\alpha_{n}^{2}}{2}\left(1+\frac{2 \alpha_{n}}{3}+\frac{2 \alpha_{n}^{2}}{4}+\ldots\right)<0$. Hence $\Sigma \beta_{n}$ converges if and only if $\Sigma\left|\beta_{n}\right|$ converges, and then the left hand side of the inequality mentioned in the above proof of Theorem 4.3 can be used, showing that $\Sigma\left|\beta_{n}\right|$ converges implies $\Sigma\left|\alpha_{n}\right|^{2}$ converges.

\section{MORE ON CONVERGENCE OF EULER PRODUCTS FOR $\sigma>.5$}

Based on the theorems in Section 4, we present further numerical evidence for the convergence of the Euler products for $\sigma>.5$, for the three Dirichlet characters considered in Section 3. Indeed, this evidence is stronger in the sense that it will apply to any $s=\sigma+i t, \sigma>.5$, whereas in Section 3 we took just one typical value $t=30$. Furthermore, it also appears to be at least as or even more convincing than the evidence in Section 3.

As seen in Section 4, the convergence of an infinite product is equivalent to that of its reciprocal, so we consider the reciprocal Euler product $L^{-1}(s, \chi)=\Pi_{p}\left(1-\chi(p) \cdot p^{-s}\right)$, taken over the primes $p$, for any Dirichlet character $\chi$. It will be convenient to write $p_{n}$ for the $n$ 'th prime, so this formula becomes

$$
L^{-1}(s, \chi)=\prod_{n=1}^{\infty}\left(1-\chi\left(p_{n}\right) \cdot p_{n}^{-s}\right)=\prod_{n=1}^{\infty}\left(1-\alpha_{n}\right)
$$

where $\alpha_{n}=\chi\left(p_{n}\right) \cdot p_{n}^{-s}$. Note that $\Sigma \alpha_{n}$ is a Dirichlet series $\Sigma a_{n} \cdot n^{-s}$ with $a_{n}=0$ at all composite numbers $n$, and $a_{p}=\chi(p)$ at a prime $p$. To show convergence of the above infinite product we seek to apply Corollary 4.4 above. Since $\left|\chi\left(p_{n}\right)\right| \in\{0,1\}$ and $\sigma>1 / 2$, the second condition of this corollary 


\section{Scaled maximum sum, $B_{n}$, vs. number of primes, $\mathbf{n}$ for Dirichlet characters $\chi_{2}^{3}, \chi_{2}^{4}$ and $\chi_{2}^{5}$}

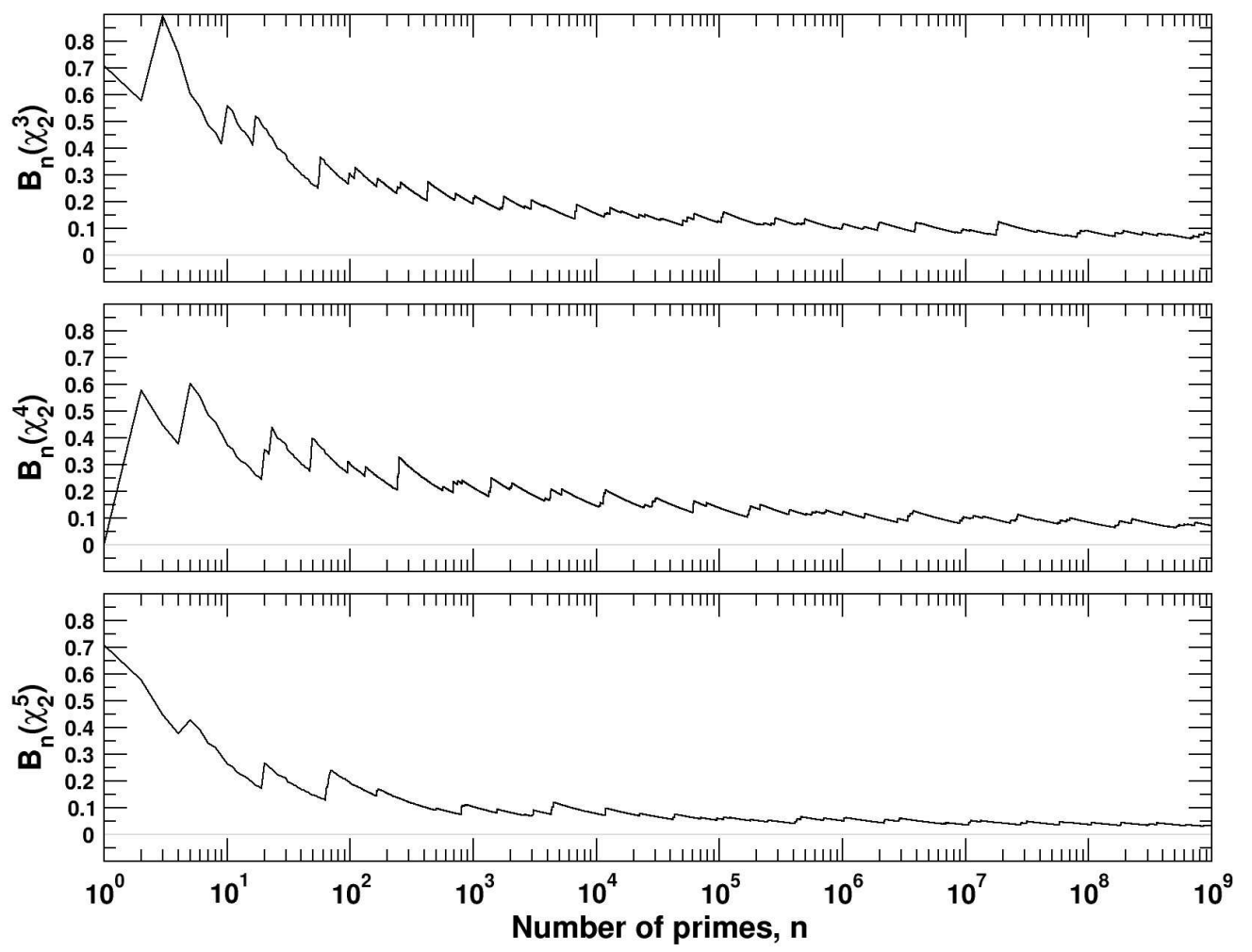

FiguRE 4. Graphs of $B_{n}$ for $\chi_{2}^{3}, \chi_{2}^{4}$, and $\chi_{2}^{5}$

is fulfilled for any Dirichlet character $\chi$. As far as the first condition $\Sigma_{n=1}^{\infty} \alpha_{n}$ convergent, we must now assume $\chi$ to be a primitive character and attempt to apply Theorem 2.12, where we now have $A_{n}=\chi\left(p_{1}\right)+\ldots+\chi\left(p_{n}\right)$. Figure 4 above and the corresponding Table 4 give strong evidence that $\left|A_{n}\right|<C \cdot p_{n}^{1 / 2}$, indeed we define $B_{n}=\max \left\{\left|A_{j}\right|\right.$ : $\left.j \leq p_{n}\right\} / \sqrt{p_{n}}$, and observe from the figure and table that $B_{n}$ is not only bounded but appears to be converging to 0. Furthermore, the following Figure 5 suggests that $B_{n}=O\left((\log (n))^{-1}\right)$. The intuition behind Figure 4 and Table 4 is that for a primitive mod $q$ character, density theorems imply that the primes in the various congruence classes $\bmod q$ occur with approximately equal frequency. This causes much cancellation in the sum for $\left|A_{j}\right|$ and therefore, even though these sums will be unbounded, they cannot grow too rapidly.

This is also related to the "prime number races," cf. [5]. Indeed, the description in Section 1 of that paper, related to the "mod 3 and $\bmod 4$ races," implies that for infinitely many primes the mod 3 race, which is between primes congruent to 1 modulo 3 and primes congruent to -1 modulo 3 , is tied. And for such primes $p_{n}$ one will have $A_{p_{n}}=0$ (for $\chi_{2}^{3}$ ). It follows that $\liminf _{n \rightarrow \infty} A_{p_{n}}=0$ for $\chi_{2}^{3}$. The situation for $\chi_{2}^{4}$ is the same (but not for $\chi_{2}^{5}$ ).

\begin{tabular}{|c|c|c|c|c|c|c|c|c|c|}
\hline \hline$n$ & 10 & $10^{2}$ & $10^{3}$ & $10^{4}$ & $10^{5}$ & $10^{6}$ & $10^{7}$ & $10^{8}$ & $10^{9}$ \\
\hline \hline$B_{n}\left(\chi_{2}^{3}\right)$ & .557 & .301 & .214 & .155 & .125 & .111 & .0938 & .0896 & .0788 \\
\hline$B_{n}\left(\chi_{2}^{4}\right)$ & .371 & .301 & .214 & .145 & .138 & .110 & .102 & .0838 & .0712 \\
\hline$B_{n}\left(\chi_{2}^{5}\right)$ & .263 & .192 & .104 & .0780 & .0587 & .0509 & .0363 & .0370 & .0319 \\
\hline \hline
\end{tabular}

TABLE 4 . Values of $B_{n}$ 


\section{Scaled maximum sum, $B_{n} \log (n)$, vs. number of primes, $\mathbf{n}$ for Dirichlet characters $\chi_{2}^{3}, \chi_{2}^{4}$ and $\chi_{2}^{5}$}

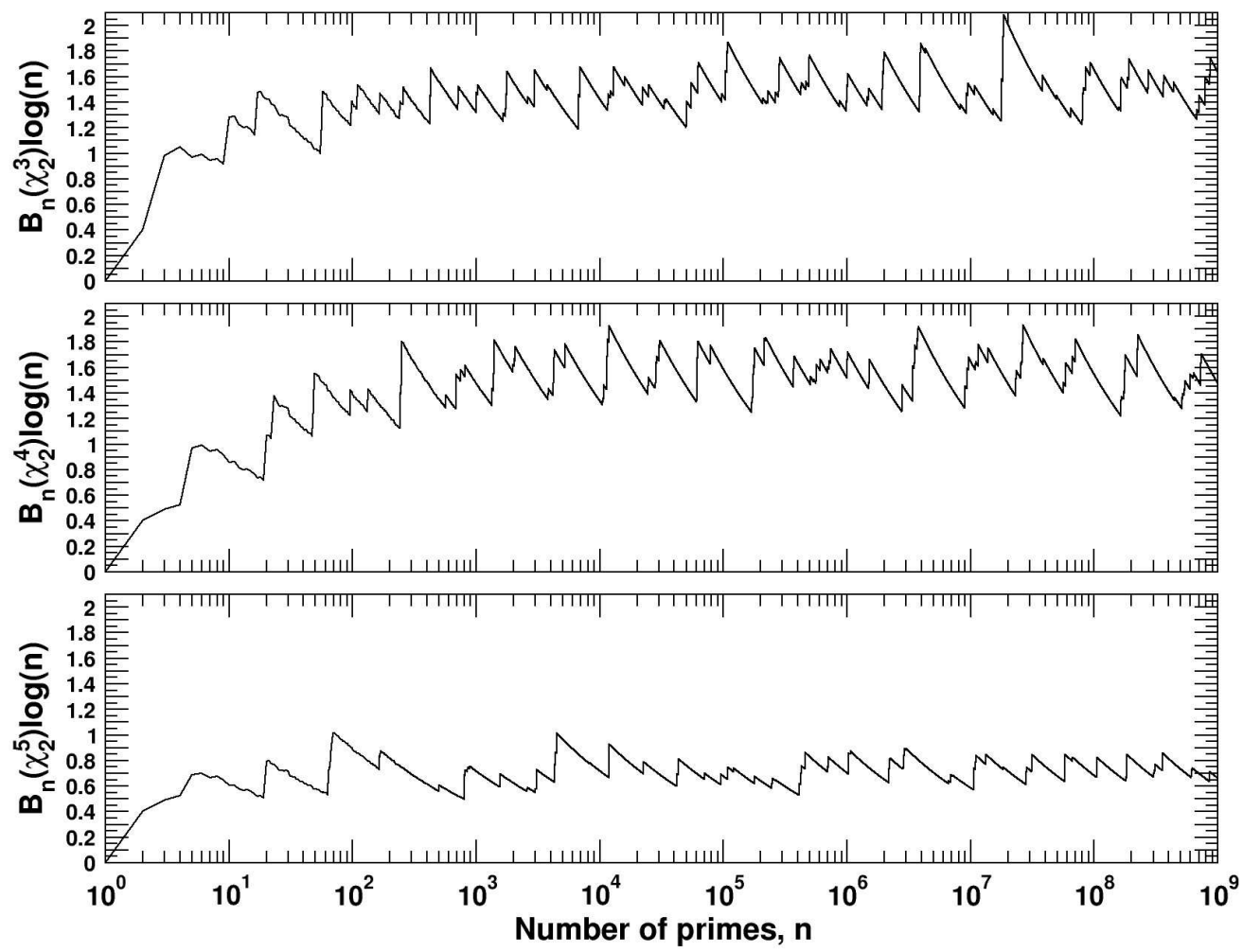

FiguRE 5. Graphs of $B_{n} \cdot \log (n)$

\section{EXAMPLES AND QUESTIONS}

Our first example is standard. Here (as before) $\Pi_{p}$ denotes a product and $\Sigma_{p}$ denotes a sum, both over the prime numbers, and $p_{n}$ is the n'th prime.

6.1 Example : The Euler product for $\zeta(s)$, or for any Dirichlet $L$-function $L(s, \chi)$, is absolutely convergent for $\sigma>1$.

To see this, e.g. for $\zeta(s)$, consider the Euler product $\zeta^{-1}(s)=\Pi_{p}\left(1-p^{-s}\right)$. Then, using the notation of Section $4, \alpha_{n}=p_{n}^{-s}$ so $\Sigma_{n} \alpha_{n}$ is clearly absolutely convergent for $\sigma>1$, and Theorem 4.1 then implies that $\zeta^{-1}(s)=\Pi_{n}\left(1-\alpha_{n}\right)$ is absolutely convergent. As seen in Section 4 this is equivalent to absolute convergence of the Euler product for $\zeta$. The proof for the $L$ - functions is similar.

The following examples will involve Theorem 4.3 (or its Corollary 4.4) and be less straightforward. As in Example 6.1 we will generally look at the inverse of the function in question for convergence, without specific mention.

6.2 Example : Let

$$
f(s)=\prod_{n \geq 2} \frac{1}{1-(-1)^{n} n^{-s}}=\frac{1}{1-2^{-s}} \cdot \frac{1}{1+3^{-s}} \cdots .
$$

Then the infinite product $f(s)$ converges for $\sigma>$ $1 / 2$.

Proof: Here $\alpha_{n}=(-1)^{n} n^{-s}, n \geq 2$. Using Theorem 2.7 shows $\Sigma_{n \geq 2} \alpha_{n}$ is convergent, $\sigma>0$. The convergence of $f(s)$ follows by Corollary 4.4 .

The next example is very similar to Example 6.2 but $n$ is replaced by $p_{n}$ so that it is an Euler-type product.

6.3 Example : Let

$$
g(s)=\prod_{n \geq 1} \frac{1}{1+(-1)^{n} p_{n}^{-s}}=
$$

$\frac{1}{1-2^{-s}} \cdot \frac{1}{1+3^{-s}} \cdot \frac{1}{1-5^{-s}} \cdot \frac{1}{1+7^{-s}} \cdot \frac{1}{1-11^{-s}} \cdots$

Then the infinite product $g(s)$ converges for $\sigma>1 / 2$. Proof: Similar to that of Example 5.2.

6.4 Examples : Euler products that arise from Dirichlet $L$-functions are further examples, and as we have seen in Section 3 and Section 5, for primitive characters, they appear to also converge for $\sigma>1 / 2$. We close this section with some potentially interesting questions.

6.5 Questions : Do the functions $f, g$ in Examples $6.2,6.3$ (particularly 6.3), also satisfy a functional equation relating the function values at $z$ and $1-z$. Are they in the Selberg class? If so they may give 
an example of a function in the Selberg class that satisfies the Riemann Hypothesis. Is there another example of this type? Thanks to theorems of Kaczoworski and Petrelli [7], and of Conrey and Ghosh [4], it is known that the Selberg class in degree $d=0$ consists of the single constant function 1, is empty for $0<d<1$, and in degree $d=1$ consists of the Riemann zeta function, together with all L-functions coming from primitive characters and their vertical translates. So $f, g$, if in the Selberg class, would have to have $d>1$.

\section{REFERENCES}

[1] T. M. Apostol, Mathematical Analysis, Second Edition, Addison-Wesley, Menlo Park CA (1974).

[2] D. H. Bailey, "MPFUN2015 : A thread-safe arbitrary precision package"

http://www.davidhbailey.com/dhbpapers/mpfun2015.pdf.
[3] R. G. Bartle, The Elements of Real Analysis, John Wiley \& Sons, N.Y., London, Sydney (1964).

[4] J. B. Conrey, A. Ghosh, On the Selberg class of Dirichlet series : small degrees, Duke Math. J. 72 (1993), 673-693.

[5] A. Granville, G. Martin, Prime number races, Amer. Math. Monthly 113, No. 1 (2006), 1-33.

[6] G. J. O. Jameson, The Prime Number Theorem. London Math. Soc., Student Texts 53, Cambridge Univ. Press, Cambridge (2003).

[7] J. Kaczoworski, A. Petrelli, On the structure of the Selberg class, $0 \leq d \leq 1$, Acta Math. 182 (1999), 207-241.

[8] S. Lang, Complex Analysis, Fourth Edition, Graduate Texts in Mathematics 103, Springer-Verlag, N.Y., Berlin (1999).

[9] Maple (17), Maplesoft, a division of Waterloo Maple Inc. Waterloo, Ontario.

[10] M. R. Murty, Problems in Analytic Number Theory, Graduate Texts in Mathematics 206, Springer-Verlag, N.Y., Berlin, Heidelberg (2001).

[11] J. P. Serre, A Course in Arithmetic, Graduate Texts in Mathematics 7, Springer-Verlag, N.Y., Berlin (1973).

[12] Wolfram Research, Inc., Mathematica, Version 9.0, Champaign, IL (2012). 


\section{КОНВЕРГЕНЦИЈА НА РЕДОВИ НА ДИРИХЛЕ И ОЈЛЕРОВИ ПРОИЗВОДИ}

Doug S. Phillips, Peter Zvengrowski

Information Technologies, University of Calgary

Department of Mathematics and Statistics, University of Calgary

Во првиот дел на овој труд се докажани теореми за конвергенција на редови на Дирихле, кои се подобрување на класичните теореми за конвергенција, како на пример во книгата „Курс по аритметика“ од Сер. Вториот дел се однесува на производи од Ојлеров тип. Докажана е теорема за конвергенција која дава доволни услови за такви производи да конвергираат во полурамнината што има реален дел поголем од $1 / 2$. Исто така изнесени се нумерички согледувања, кои сугерираат дека Ојлеровите производи кои што

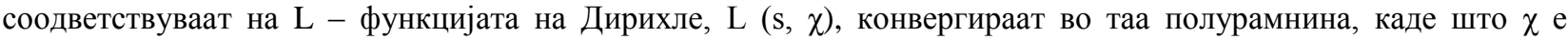
примитивниот карактер на Дирихле.

Клучни зборови: Редови на Дирихле, Ојлерови производи, L-функции, примитивен карактер на Дирихле 